This item was submitted to Loughborough's Research Repository by the author.

Items in Figshare are protected by copyright, with all rights reserved, unless otherwise indicated.

\title{
Data association using game theory for multi-target tracking in passive
}

\section{bistatic radar}

PLEASE CITE THE PUBLISHED VERSION

https://doi.org/10.1109/RADAR.2017.7944168

PUBLISHER

(C) IEEE

VERSION

AM (Accepted Manuscript)

LICENCE

CC BY-NC-ND 4.0

\section{REPOSITORY RECORD}

Daniyan, Abdullahi, Abdulrazaq Aldowesh, Yu Gong, and Sangarapillai Lambotharan. 2019. "Data Association Using Game Theory for Multi-target Tracking in Passive Bistatic Radar". figshare.

https://hdl.handle.net/2134/37350. 


\title{
Data Association Using Game Theory for Multi-target Tracking in Passive Bistatic Radar
}

\author{
Abdullahi Daniyan, Abdulrazaq Aldowesh, Yu Gong, and Sangarapillai Lambotharan
}

\begin{abstract}
We investigate a game theoretic data association technique for multi-target tracking (MTT) with varying number of targets in a real passive bi-static radar (PBR) environment. The radar measurements were obtained through a PBR developed using National Instrument (NI) Universal Software Radio Peripheral (USRP). We considered the problem of associating target stateestimates-to-tracks for varying number of targets. We use the sequential Monte Carlo probability hypothesis density (SMC-PHD) filter to perform the multi-target tracking in order to obtain the target state estimates and model the interaction between target tracks as a game. Experimental results using this real radar data demonstrate effectiveness of the game theoretic data association for multiple target tracking.

Index Terms-Game theory, data association, multi-target tracking, passive bi-static radar PBR, particle filter, sequential Monte Carlo (SMC), PHD filter.
\end{abstract}

\section{INTRODUCTION}

Data association deals with the problem of assigning measurements-to-target or target-state-estimates to individual target tracks [1], [2]. The measurement-to-target approaches involve using explicit data association techniques to assign measurements to each target track [3] and then applying a single target tracker [1], [4], [5] for example the Kalman filter (KF) or the particle filter (PF) to perform tracking using the assigned measurement. Target-state-estimate methods require using multitarget tracking (MTT) algorithms to first perform multi-target tracking to obtain instantaneous target-state-estimates followed by data association techniques to assign the estimates to various tracks. The data association problem become more challenging in MTT in the case of missed detection and false alarms in measurements and especially when the number of targets vary with time.

Techniques often used to address the MTT data association problem include the nearest neighbour (NN) algorithm [6], the joint probabilistic data association (JPDA) filter [7], [8] and the multiple hypothesis tracker (MHT) [9]. These algorithms are more suited to linear update/linear measurement and Gaussian uncertainty scenarios [10]. However, in complex MTT scenarios the target dynamics can be non-linear and even non-Gaussian. For such scenarios, [10] and [11] proposed a measurementto-track data association for MTT using game theory. In their approach, a known and fixed number of targets was considered

\footnotetext{
This work was supported by the Engineering and Physical Sciences Research Council (EPSRC) Grant number EP/K014307/1, the MOD University Defence Research Collaboration (UDRC) in Signal Processing, UK and the Petroleum Technology Development Fund (PTDF), Nigeria.

A. Daniyan, Y. Gong and S. Lambotharan are with the Signal Processing and Networks Research Group, Wolfson School of Mechanical, Electrical and Manufacturing Engineering, Loughborough University, UK (\{a.daniyan, y.gong, s.lambotharan\}@lboro.ac.uk).

A. Aldowesh is with Prince Sultan Advanced Tech. Research Institute (PSATRI), King Saud University, Riyadh, Saudi Arabia (dh_000@hotmail.com).
}

and the aim was to use game theory to assign measurements to individual single target trackers. This approach however is constrained because the number of targets need to be known. Recently, we proposed a game theoretic data association technique for MTT with varying number of targets [12]. In our approach, the various target tracks were the set of player and we formulated their interaction as a game. We defined the utility function of this game and used the instantaneous target-state-estimates as the strategy set. A regret matching algorithm with forgetting factor was used to find the equilibrium of the game. We demonstrated the performance of this technique in [12] using simulated data.

One common algorithm used to perform MTT is the probability hypothesis density (PHD) filter. The PHD filter is a recursion that propagates the first order moment of the random finite set (RFS) of targets in time [5], [13]. The PHD filter is able to track time varying multi-targets without the need to explicitly associate measurements to tracks. The PHD filter has been implemented in two distinct fashions; i.e. as the Gaussian mixture PHD (GM-PHD) filter [14] and the sequential Monte Carlo PHD (SMC-PHD) filter [15]. In the SMC-PHD filter implementation, the PHD is approximated by a set of weighted particles and therefore more suitable for tracking in non-linear and non-Gaussian environments.

In this paper, we demonstrate the performance of our proposed game theoretic data association (GTDA) technique in [12] on real data. In order to achieve this, we set-up a passive bistatic radar (PBR) system using the NI USRP software defined radio platform with necessary hardware to record detections from moving aeroplanes. We applied a multi-target tracker (the SMC-PHD filter) to the recorded PBR data to track aeroplanes detected during the recording and obtained target-state-estimates. To find target state-estimate-to-track associations, we formulate the problem of data association as a game between multiple and varying number of tracks in the same manner as in [12]. We applied the GTDA to the tracked targets in the PBR recording to perform target-state-estimate to track data association.

\section{The Passive Bi-static Radar System}

In this section, we discuss some key steps in setting up our PBR system for the detection of moving aerial targets (e.g. aeroplanes).

\section{A. System Architecture}

The PBR system implementation relies on exploiting a software-defined radio platform (SDR). The computationally demanding part of the passive radar signal processing chain is implemented on the software-defined radio FPGA, and the rest of the radar processing chain, such as target detection, multitarget tracking and data association have been implemented on the host CPU, (see Fig. 1). 


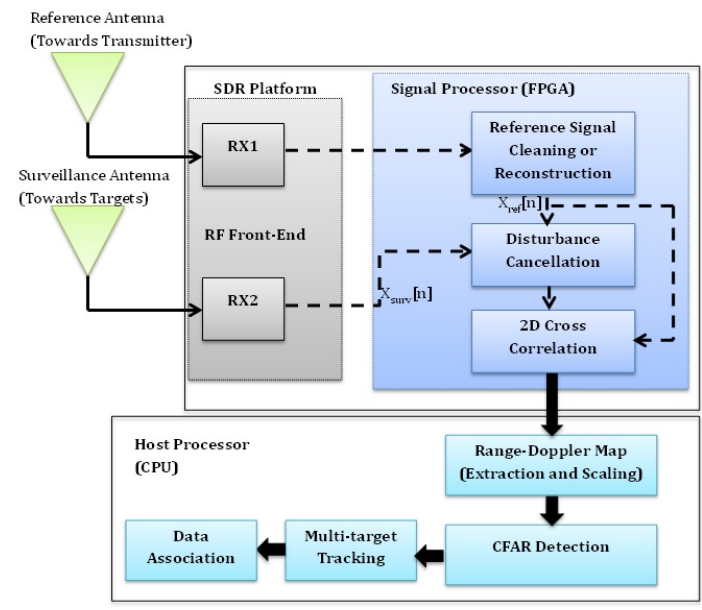

Figure 1: PBR System Architecture.

\section{B. Signal reconstruction and disturbance cancellation}

The SDR platform is the NI-USRP-2950R, and contains two $\mathrm{RF}$ channels that can be configured as two synchronized receivers that provide the reference signal and surveillance signal. The antenna for the reference signal has been pointed towards the direction an FM transmitter. Since the surveillance antenna will not completely suppress the strong direct signal, a disturbance cancellation operation is performed on the surveillance signal using least mean square (LMS) adaptive algorithm. After that, the surveillance and reference signals have been used to perform the two-dimensional cross-correlation function (2D-CCF) to obtain range -Doppler map.

\section{Two-Dimensional Cross-Correlation Function (2D-CCF)}

The evaluation of the bi-static range-Doppler $2 \mathrm{D}-\mathrm{CCF}$ is the key step in the PBR processing chain. It corresponds to the implementation of a bank of matched filters, each one tuned to a specific target bi-static Doppler frequency shift.

$$
\chi(u, m)=\sum_{n=0}^{N-1} x_{\text {surv }}[n] x_{r e f}^{*}[n-u] e^{-j 2 \pi \frac{m n}{N}}
$$

where $x_{r e f}^{*}[u]$ denotes the sampled reference signal, $x_{\text {surv }}^{*}[u]$ denotes the sampled surveillance signal and $\chi(u, m)$ denote the 2D-CCF. $N$ is Number of integrated samples, $u$ is the time bin corresponding to time delay $\tau=\frac{u}{f_{s}}$; where $f_{s}$ is sampling rate and $m$ is Doppler bin corresponding to Doppler shift $f_{d}=\frac{m f_{s}}{N}$.

The 2D-CCF stage serves two important purposes: the generation of sufficient signal processing gain to allow the targets to be detected above the noise floor and the estimation of the bi-static range and Doppler shift of the target echoes.

\section{Target Detection (Order Statistic CFAR)}

Having calculated the 2D-CCF correlation surface, target detection is performed by comparing the magnitude of each range Doppler bins to a threshold. The threshold for different range Doppler bins will vary according to the estimate of disturbance and noise for each range - Doppler bin in order to maintain a constant false alarm rate. The Constant false alarm rate (CFAR) algorithm of choice was the order statistic OS-CFAR [17] and [16], for its robustness against interfering peaks on the cell under test (CUT). Fig. 2 shows the algorithm we used for implementing

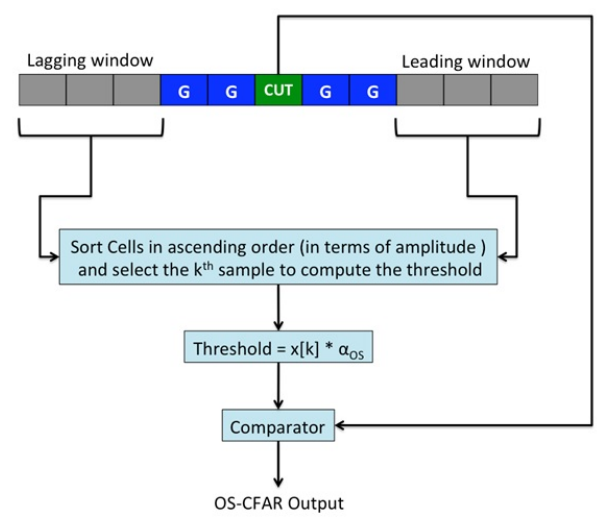

Figure 2: Order statistic CFAR Algorithm [16].

the OS-CFAR. Cells before and after a CUT are utilized to estimate the noise floor level around the CUT, by arranging them in ascending order in terms of their amplitude value, and taking only the $k$ th sample to compute the threshold for a specific probability of false alarm $\left(P_{F A}\right)$. Guard cells before and after the CUT are needed in case the energy of the CUT is dispersed onto adjacent cells. The threshold for determining whether a target exist in the CUT is computed for a specific $P_{F A}$ using the following equation [17]:

$$
P_{F A}=\frac{N !\left(\alpha_{O S}+N-k\right) !}{(N-k) !\left(\alpha_{O S}+N\right) !} .
$$

where $\alpha_{O S}$ is the OS scaling factor, $k$ is the representative sample rank and $N$ is the total number of background samples.

\section{TRacking And Data Association}

This section presents both the MTT and data association models used for tracking aeroplanes in our PBR system.

\section{A. The MTT Tracking Filter}

1) State and Measurement Models: Let the constant velocity state evolution model of a target be:

$$
\hat{\boldsymbol{x}}_{k}=\boldsymbol{F} \hat{\boldsymbol{x}}_{k-1}+\boldsymbol{w}_{k}
$$

with

$$
\boldsymbol{F}=\left[\begin{array}{cc}
1 & \delta t \\
0 & 1
\end{array}\right]
$$

where $\boldsymbol{F}$ is the system transition function, $\hat{\boldsymbol{x}}_{k}=\left[\hat{r}_{k}, \hat{v}_{k}\right]^{\bar{T}}, \hat{r}_{k}$ and $\hat{v}_{k}$ denotes the bi-static range and bi-static radial velocity respectively, and $\boldsymbol{w}_{k}$ is an independent and identically distributed (i.i.d.) process noise vector. Then the multi-target state at time $k$ can be written as $\boldsymbol{X}_{k}=\left\{\hat{\boldsymbol{x}}_{1, k}, \ldots, \hat{\boldsymbol{x}}_{M, k}\right\} \in E_{s}$ where $M$ is the number of targets present at time $k$, and $E_{s}$ denotes the state space.

Let the target-originated measurement model be:

$$
\hat{\boldsymbol{z}}_{k}=\mathbf{H}\left[\begin{array}{l}
\hat{r}_{k} \\
\hat{v}_{k}
\end{array}\right]+\hat{\boldsymbol{n}}_{k}
$$

where $\mathbf{H}$ is the transformation matrix, and $\hat{\boldsymbol{n}}_{k}$ is an i.i.d. process noise vector. Measurements consist of both targetoriginated measurements and false alarms. The multi-target measurement set at time $k$ in the observation space is: $\boldsymbol{Z}_{k}=$ $\left\{\hat{\boldsymbol{z}}_{1, k}, \ldots, \hat{\boldsymbol{z}}_{a, k}\right\} \bigcup\left\{\mathbf{c}_{1, k}, \ldots \mathbf{c}_{b, k}\right\} \in E_{o}$ where $\left\{\mathbf{z}_{1, k}, \ldots, \mathbf{z}_{\alpha, k}\right\}$ denotes the target-originated measurement set with number of 


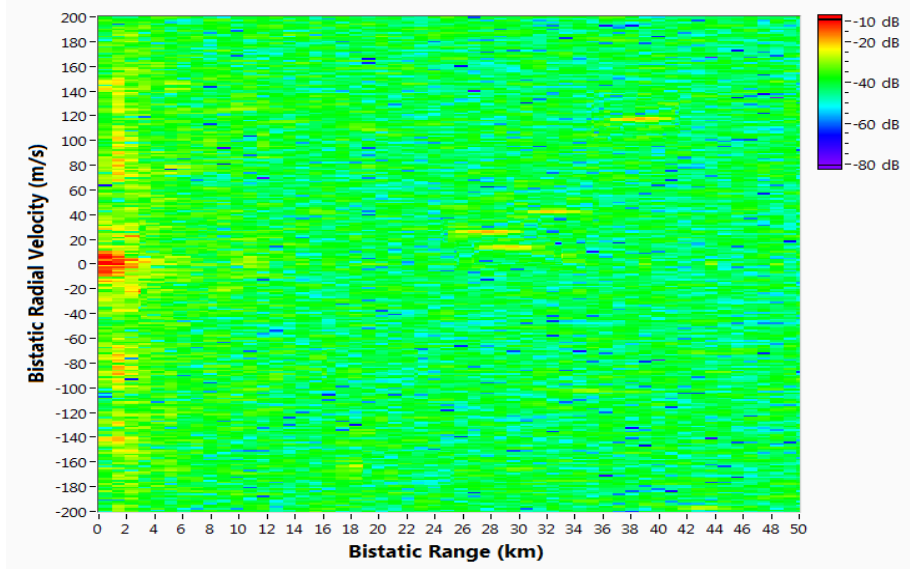

(a) Bi-static radial velocity vs bi-static range intensity map before CFAR.

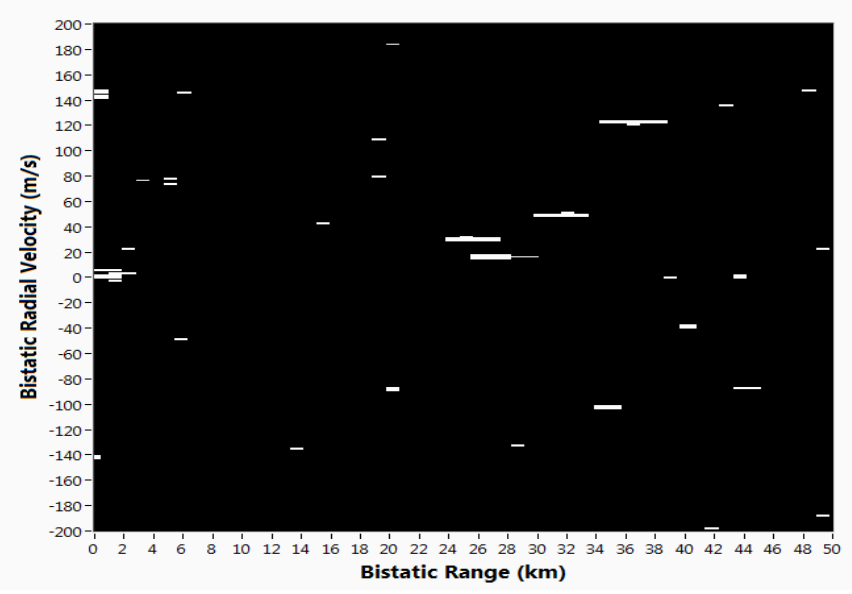

(b) Bi-static radial velocity vs bi-static range map after CFAR.

Figure 3: Bi-static radial velocity vs bi-static range map obtained from the passive radar set-up at time $=109 \mathrm{~s}$.

measurements, $\alpha ;\left\{\mathbf{c}_{1, k}, \ldots \mathbf{c}_{\beta, k}\right\}$ denotes the false measurement set with the number of measurements, $\beta$ and $E_{o}$ denotes the observation space. Then the multi-target cumulative measurement set at time $K$ is $\boldsymbol{Z}_{1: K}=\left\{\mathbf{Z}_{1}, \mathbf{Z}_{2}, \ldots, \mathbf{Z}_{K}\right\} \in E_{o}$.

\section{B. SMC-PHD Filtering}

We implement the SMC-PHD filter in the same manner as in [12] using the above state and measurement models to obtain the target-state-estimates as the output of the SMC-PHD filter.

Therefore at time $k$, the output of the SMC-PHD filter is a set of target state estimates given as: $\tilde{\boldsymbol{x}}_{k}=\left\{\tilde{\boldsymbol{x}}_{1, k}, \tilde{\boldsymbol{x}}_{2, k}, \cdots, \tilde{\boldsymbol{x}}_{T_{k}, k}\right\}$, where $\tilde{\boldsymbol{x}}_{t, k}=\left[\hat{r}_{k}, \hat{v}_{k}\right]^{\bar{T}}, \hat{r}_{k}$ and $\hat{v}_{k}$ denotes the bi-static range and bi-static radial velocity respectively. It is assumed that, each target, when present generates at most one measurement.

\section{The Game}

We use the game theoretic data association (GTDA) technique with regret matching and forgetting factor [12] to perform targetstate-estimate to track association for our PBR system. At time $k$, the players, $\mathcal{P}_{k}$ are the tracks for different targets and can vary from time to time depending on the estimated number of targets by the SMC-PHD filter, $T_{k}$. However, at each time instance, the number of players is finite. Each player wants to assign a new target-state-estimate (the strategies) to its existing track. The strategy set $\mathcal{S}_{k}=\left\{s_{k}^{i}\right\}_{i=1}^{T_{k}}$ of each player corresponds to the set of target state estimates from the SMC-PHD filter and they are known to all of the players at time $k$. The utility function, $u_{p, k}\left(s_{p, k}, s_{-p, k}\right)$ is defined based on a statistical distance and feasibility measure, see [12] such that $\mathcal{S} \longrightarrow \mathbb{R}$, where $s_{p, k}$ denote the action of the $p$ th player at time $k,-p_{k}$ refers to all players except player $p_{k}$.

At each time instant, a player may either continue to play the same strategy as in the previous time instant, or switch to other strategies, with probabilities that are proportional to how much its regret would have been had it always made that change in the past. Specifically, let $s_{p, k} \in \mathcal{S}_{k}$ denote the strategy of the $p$ th player in the $k$ th iteration and let the index of $s_{p, k}$ within $\mathcal{S}_{k}$ be $n$ such that $n \in\left\{1, \cdots, T_{k}\right\}$. Also, let $s_{p, k-1} \in \mathcal{S}_{k-1}$ be the strategy of the $p$ th player at the $k-1$ iteration and let $s_{p, k-1}$ have index $l$ such that $l \in\left\{1, \cdots, T_{k-1}\right\}$. Each player computes the average regret for choosing the $n$th strategy in the $k$ th iteration using:

$$
\tilde{r}_{p, n}(k)=\max \left\{0, \tilde{R}_{p, n}(k)\right\}
$$

$$
\begin{aligned}
& \tilde{R}_{p, n}(k)= \\
& \frac{1}{k-1} \sum_{l=1}^{k-1} \lambda_{f}^{(k-1)-l}\left[u_{p, k}\left(n, s_{-p, k}(l)\right)-u_{p, k}\left(l, s_{-p, k}(l)\right)\right]
\end{aligned}
$$

where $\tilde{R}_{p, n}(k)$ represent the average pay-off (with exponential forgetting factor) for the $p$ th player at time $k$ for not having played a different strategy $n$ each time the strategy $l$ was played in the past; $0<\lambda_{f} \leq 1$ is the forgetting factor. Each player then chooses the $n$th strategy that satisfies

$$
\arg \max _{n} \beta_{p, n}(k)
$$

where the distribution $\beta_{p, n}(k)$ is given by

$$
\beta_{p, n}(k)= \begin{cases}\frac{1}{\alpha} \tilde{r}_{p, n}(k), & \text { if } l \neq n \\ 1-\sum_{\substack{n \in\left\{0,1, \cdots, T_{k}\right\} \\ n \neq l}} \beta_{p, n}(k), & l=n .\end{cases}
$$

The constant $\alpha>0$ is a large enough number such that $\beta_{p, n}(k)>0$ and this ensures that there is always a positive probability of playing the same strategy as in the previous step [18]. When a new player, $p^{+}$joins the game, i.e. when $T_{k}>T_{k-1}$, its own distribution, $\beta_{p^{+}}(k)$ is started such that $\beta(k)=\cup \beta_{p^{+}}(k)$ at that instance. In regret matching, the correlation in the plays of different players arises from the commonly observed history. Thus, the history serves as a signal in giving the private recommendation to each player [18]. For more details on the GTDA technique, the reader is referred to [10], [12].

\section{EXPERIMENTAL RESULTS}

\section{A. Experimental Set-up}

In this section, we applied the GTDA technique with exponential forgetting factor proposed in [12] in a real passive radar 


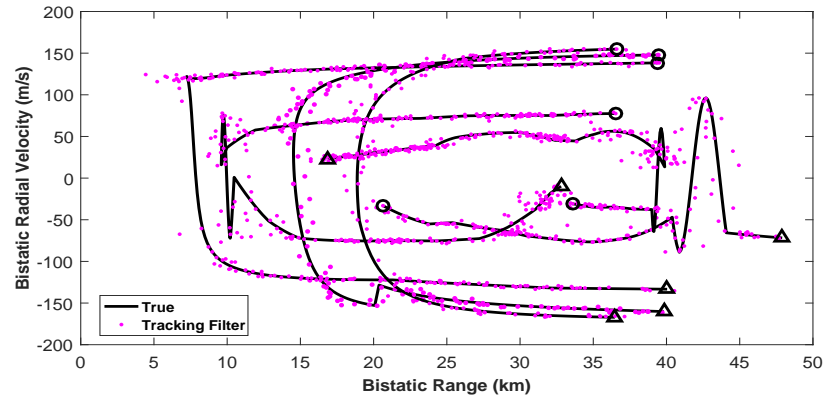

(a)

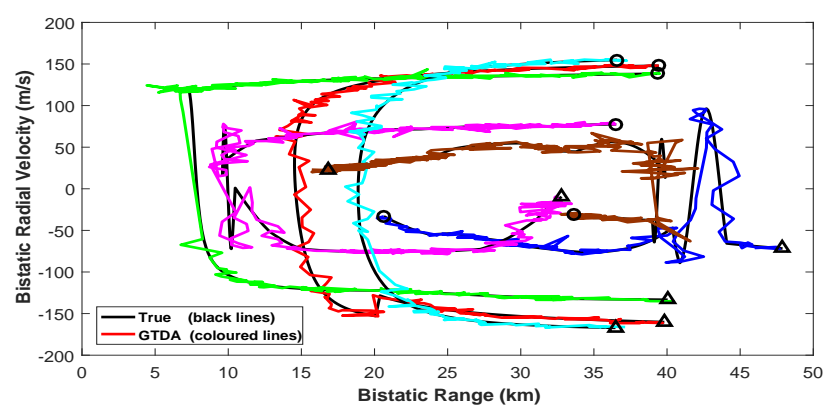

(c)

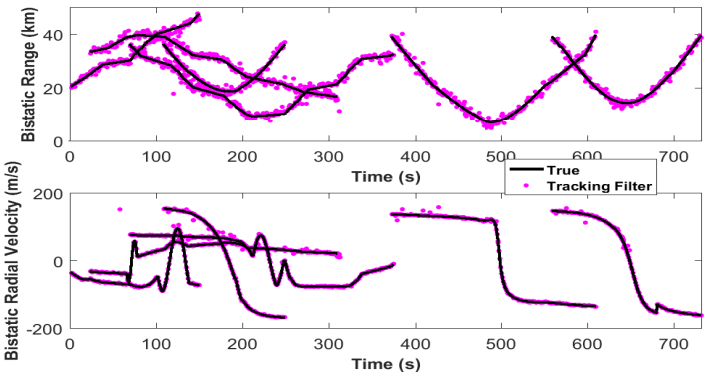

(b)

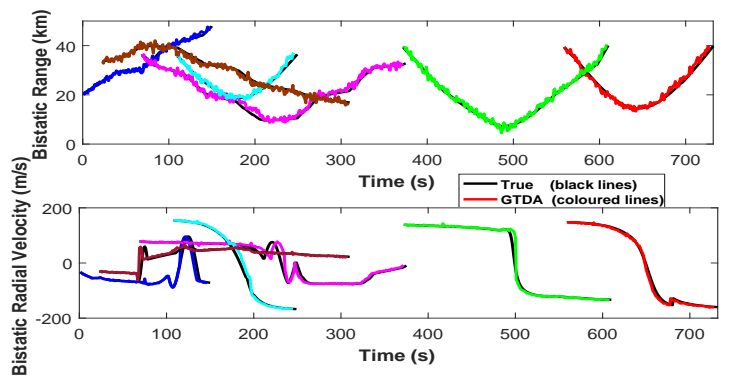

(d)

Figure 4: Tracking filter and GTDA plots: (a) Bi-static radial velocity vs bi-static range plot showing various true flight paths and tracking filter estimates for the whole duration of the experiment. Start/End for each target are shown with $\bigcirc / \triangle$; (b) Tracking filter estimates superimposed on true flight positions in both bi-static range and bi-static radial velocity axis (versus time); (c) Bi-static radial velocity vs bi-static range plot showing various true flight paths and GTDA data association for the whole duration of the experiment. Start/End for each target are shown with $\bigcirc / \triangle$; (d) True flight positions in both bi-static range and bi-static radial velocity axis and GTDA data association (versus time) showing target entry and exit.

experimental setting. The goal is to track aeroplanes using bistatic range and radial velocity measurements obtained from a passive radar set-up and then associate the target-state-estimates to the various aeroplane tracks using the GTDA technique. The experiment was carried out on the 7th of July, 2016 on top the roof of Sir David Davies Building, Loughborough University, UK.

In this experiment, the transmitter of opportunity is an FM transmitter with a center frequency of $106 \mathrm{MHz}$ and located some $6.5 \mathrm{~km}$ away from the receiver. The passive receive end consists of a National Instruments SDR platform (the NI-USRP2950R) and two omni-directional FM antennas; one is used for the surveillance channel and the other is used for the reference channel. We interfaced the SDR platform with LabView to record the raw in-phase and quadrature (I-Q) data for a duration of 12 mins 12 secs and processed to obtain the bi-static range and radial velocity measurements of planes within the surveillance scene. The receiver had a bi-static range resolution of $937.5 \mathrm{~m}$ and bi-static radial velocity resolution of $1.4 \mathrm{~m} / \mathrm{s}$. The system was set to have a maximum detection range of approximately $48 \mathrm{~km}$ and only detections above an altitude of $100 \mathrm{~m}$ were considered. During the duration of the recording, a total of six aeroplanes entered and exited the detectable region of the receiver. The processed passive radar data were recorded as an intensity map with the bi-static radial velocity (in $\mathrm{m} / \mathrm{s}$ ) on the $\mathrm{y}$-axis and the bi-static range (in $\mathrm{km}$ ) on the $\mathrm{x}$-axis. CFAR detection was then applied to the raw intensity map measurements to obtain a binary intensity map to facilitate tracking. The probability of false alarm used for the CFAR was $1 \times 10^{-4}$. The intensity maps of the bi- static range and radial velocity measurements recorded at time $=109 \mathrm{~s}$ before and after applying CFAR are shown in Fig. 3a and Fig. $3 b$ respectively. In Fig. 3b, the colour white indicates detections and black indicates no detection. It is observed from Fig. $3 b$ that the detections are smeared therefore, centroiding was performed to obtain single point detection before being passed to the tracking filter.

The tracking filter (the SMC-PHD filter) takes in the centroided CFAR detections as measurements to perform MTT and output target-state-estimates. We model the process noise in (3), $\boldsymbol{w}_{k}$ to be distributed according to $\mathcal{N}\left(0, \sigma^{2} \boldsymbol{Q}\right)$, where $\sigma=0.02 \mathrm{~km} / \mathrm{s}^{2}$, the sampling period $\delta t=1 s$ and

$$
\boldsymbol{Q}=\left[\begin{array}{ll}
\frac{\delta t^{4}}{4} & \frac{\delta t^{3}}{2} \\
\frac{\delta t^{3}}{2} & \delta t^{2}
\end{array}\right] .
$$

The measurement noise in (4) was modelled as $\hat{\boldsymbol{n}}_{k}=\mathcal{N}(0, \hat{\boldsymbol{R}})$ where $\hat{\boldsymbol{R}}=\operatorname{diag}\left(\left[\sigma_{\hat{r}}^{2}, \sigma_{\hat{v}}^{2}\right]\right)$ with $\sigma_{\hat{r}}=30 \mathrm{~m}$ and $\sigma_{\hat{v}}=4.5 \mathrm{~m} / \mathrm{s}$; where $\mathbf{H}=\operatorname{diag}\left([1,1]^{\bar{T}}\right)$.

\section{B. Results}

Fig. 4a and Fig. 4b show results obtained from one sample run of the SMC-PHD filter on the passive radar detections for the whole duration of the experiment. The number of particles per existing target was set to $\rho=4000$ and the number of particles for new born tracks was set to $\frac{\rho}{5}$. In Fig. $4 \mathrm{a}$, solid black lines represent the true flight paths on the bi-static range and radial velocity map, while the dots represent the filter estimates. The circle and triangle denote the start and end measurements of the flights. The true flight bi-static range and radial velocity data 
were obtained from [19]. Notice from Fig. 4a that there are a total of six targets throughout the duration of the experiment. Three of the targets having a " $C$ " like trajectory correspond to targets passing over the transmit/receive set-up of the passive radar. Hence, the upper part of the "C" shape corresponds to approaching targets and thus have positive bi-static radial velocity and the lower part of the "C" shape corresponds to receding targets having a negative bi-static radial velocity. A zero bi-static radial velocity corresponds to when targets are closest in bi-static range to the transmit/receive set-up. The other three targets having irregular trajectories correspond to targets taking position after having taken off or taking position to land at an airport within the detection range of the receiver. In Fig. 4b, the bi-static range and radial velocity of the true flight path (solid lines) and the tracking filter estimates (dots) versus time are shown. From this figure the time each target enters and exits the tracking scene can be observed.

In Fig. 4c, the bi-static range and radial velocity of the true flight path (black) and the target-state-estimate after GTDA (coloured) of [12] are shown. In Fig. 4d, the bi-static range and radial velocity of the true flight path (black) versus time and the target-state-estimate after GTDA (coloured) of [12] are shown. These results suggest that the proposed GTDA technique of [12] is able to properly associate the target-state-estimates of different targets to their corresponding tracks.

\section{CONCLUSION}

We demonstrated effectiveness of recently proposed game theoretic data association method for multiple targets tracking using real field measurements collected by an NI-USRP based PBR system. SMC-PHD filter was used to track aeroplanes and GTDA was used for track association. Experimental results demonstrates that the GTDA technique is able to successfully associate the target-state-estimates to various target tracks.

\section{REFERENCES}

[1] Y. Bar-Shalom, P. Willett, and X. Tian, Tracking and Data Fusion: A Handbook of Algorithms. YBS Publishing, 2011.

[2] Y. Bar-Shalom, "Multitarget-multisensor tracking: advanced applications," Norwood, MA, Artech House, 1990, 391 p., vol. 1, 1990.

[3] Y. Bar-Shalom, X. R. Li, and T. Kirubarajan, Estimation with Applications to Tracking and Navigation: Theory Algorithms and Software. John Wiley \& Sons, 2004.

[4] R. P. Mahler, Statistical multisource-multitarget information fusion. Artech House, Inc., 2007.

[5] R. Mahler, "Statistics 102 for multisource-multitarget detection and tracking," IEEE Journal of Selected Topics in Signal Processing, vol. 7, no. 3, pp. 376-389, 2013.

[6] S. Blackman and R. Popoli, Design and Analysis of Modern Tracking Systems, ser. Artech House radar library. Artech House, 1999.

[7] T. E. Fortmann, Y. Bar-Shalom, and M. Scheffe, "Multi-target tracking using joint probabilistic data association," in 19th IEEE Conference on Decision and Control including the Symposium on Adaptive Processes. IEEE, 1980, pp. 807-812.

[8] J. A. Roecker and G. L. Phillis, "Suboptimal joint probabilistic data association," IEEE Transactions on Aerospace and Electronic Systems, vol. 29, no. 2, pp. 510-517, Apr 1993.

[9] S. S. Blackman, "Multiple hypothesis tracking for multiple target tracking," IEEE Aerospace and Electronic Systems Magazine, vol. 19, no. 1, pp. 5-18, Jan 2004.

[10] P. Chavali and A. Nehorai, "Concurrent particle filtering and data association using game theory for tracking multiple maneuvering targets," IEEE Transactions on Signal Processing, vol. 61, no. 20, pp. 4934-4948, 2013.

[11] _ - "Distributed data association for multiple-target tracking using game theory," in Radar Conference (RADAR), 2013 IEEE, April 2013.

[12] A. Daniyan, Y. Gong, and S. Lambotharan, "Game theoretic data association for multi-target tracking with varying number of targets," in 2016 IEEE Radar Conference (RadarConf), May 2016.
[13] R. P. S. Mahler, "Multitarget Bayes filtering via first-order multitarget moments," IEEE Transactions on Aerospace and Electronic Systems, vol. 39, no. 4, pp. 1152-1178, Oct 2003.

[14] B.-N. Vo and W.-K. Ma, "The Gaussian mixture probability hypothesis density filter," IEEE Transactions on Signal Processing, vol. 54, no. 11, pp. 4091-4104, 2006.

[15] B.-N. Vo, S. Singh, and A. Doucet, "Random finite sets and sequential Monte Carlo methods in multi-target tracking," in Proceedings of the International Radar Conference, 2003., 2003, pp. 486-491.

[16] S. Blake, "OS-CFAR theory for multiple targets and nonuniform clutter," IEEE Transactions on Aerospace and Electronic Systems, vol. 24, no. 6, pp. 785-790, Nov 1988.

[17] H. Rohling, "Radar cfar thresholding in clutter and multiple target situations," IEEE Transactions on Aerospace and Electronic Systems, vol. AES-19, no. 4, pp. 608-621, July 1983.

[18] S. Hart and A. Mas-Colell, "A simple adaptive procedure leading to correlated equilibrium1," Econometrica, vol. 68, no. 5, p. 11271150, 2000.

[19] Flightradar24. (2016) Flightradar24.com - live flight tracker! [Online]. Available: https://www.flightradar24.com 